\title{
Análise multicriterial para avaliação da governança dos recursos hídricos nas bacias dos Rios Piracicaba, Capivari e Jundiaí

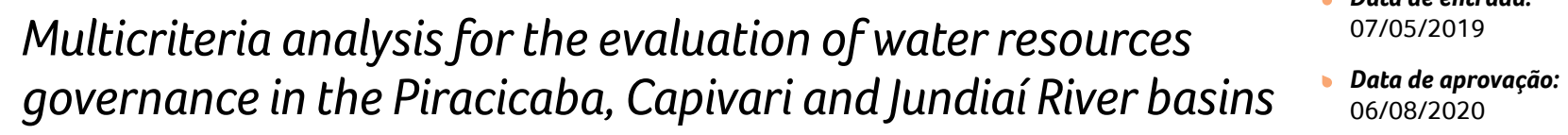

Diego Lino da Luz ${ }^{1 *} \mid$ Ayri Saraiva Rando ${ }^{1}$ | André Munhoz de Argollo Ferrão ${ }^{1}$ | Antonio Carlos Zuffo' ${ }^{1}$

DOI: https://doi.org/10.36659/dae.2022.004

ORCID ID

Luz DL (iD https://orcid.org/0000-0003-1048-5585

Rando AS iD https://orcid.org/0000-0002-0015-6334
Ferrão AMA (iD https://orcid.org/0000-0003-0687-3622 Zuffo AC (D) https://orcid.org/0000-0002-2186-9755

\section{Resumo}

No Brasil, as dificuldades referentes à avaliação da governança dos sistemas de gerenciamento dos recursos hídricos são evidentes, entre elas a seleção de critérios e subcritérios para medição dos níveis de tal governança no âmbito das unidades de gerenciamento dos recursos em questão. $O$ objetivo deste artigo é aplicar a análise multicriterial para avaliação e medição dos níveis de governança do sistema de gerenciamento dos recursos hídricos nas bacias hidrográficas dos rios Piracicaba, Capivari e Jundiaí, no período entre 2013 e 2017. Para isso, tal análise utiliza os seguintes modelos ou métodos: Programação por Compromisso, Teoria dos Jogos Cooperativos, Método Analítico Hierárquico, ELECTRE II e PROMETHE II. Os critérios e subcritérios analisados foram definidos em entrevistas com especialistas ligados diretamente a essa temática, sendo que a atribuição de pesos aos mesmos ocorreu por meio da técnica DELPHI. Os resultados mostram o ano de 2017 como a melhor alternativa em relação ao nível de desempenho de governança nas bacias mencionadas.

Palavras-chave: Análise multicriterial. Modelos de auxílio à decisão. Governança nas Bacias PC].

\section{Abstract}

In Brazil, the difficulties regarding the evaluation of governance of water resources management systems are evident, among them the selection of criteria and subcriteria for measuring the levels of such governance within the management units in question. The objective of this article is to apply the multicriteria analysis to evaluate and measure the levels of governance of the management system of these resources in the Piracicaba, Capivari and Jundiai river basins, between 2013 and 2017. Such analysis uses the following models or methods: Compromise Programming, Cooperative Game Theory, Analytic Hierarchy Process, ELECTRE II and PROMETHE II. The criteria and subcriteria analyzed were defined in interviews with specialists directly related to this subject, and the attribution of weights to them occurred through the DELPHI technique. The results show the year 2017 as the best alternative in relation to the level of governance performance in the mentioned basins.

Keywords: Multicriteria analysis. Decision support models. PCJ river basis governance.

\footnotetext{
1 Universidade Estadual de Campinas (UNICAMP) - Campinas - São Paulo - Brasil.

* Autor correspondente: diego.luzagmail.com.
} 


\section{INTRODUÇÃO}

A necessidade e relevância da avaliação e monitoramento de políticas públicas são reconhecidas, mas a avaliação da governança do Sistema Nacional de Gerenciamento dos Recursos Hídricos não é executada. Entre as dificuldades para medição dos níveis de governança desse sistema estão a seleção de critérios e subcritérios (adequados para avaliação no âmbito das Unidades de Gerenciamento dos Recursos Hídricos - UGRHIs) que considerem as diferenças e peculiaridades regionais, bem como as próprias coleta e análise dos dados correlatos.

Existem estudos que propõem o uso de dimensões, aspectos e indicadores para avaliação da governança do sistema nacional, porém a coleta de dados para concretização da primeira avaliação encontra inúmeros obstáculos. Existem também métodos ou modelos de auxílio à tomada de decisão que apoiam a compreensão aprofundada de problemas ambientais, de gerenciamento dos recursos citados e de saneamento, promovendo debates em torno de tais problemas e suporte para decisão mais adequada por parte dos gestores e especialistas.

Diante da situação exposta, precisam-se levar em consideração as diferenças e peculiaridades mencionadas, além de aproximar o olhar para as unidades de gerenciamento citadas, neste caso, a Unidade de Gerenciamento dos Recursos Hídricos das Bacias dos Rios Piracicaba, Capivari e Jundiaí- UGRHI 05.

O objetivo deste artigo é aplicar a análise multicriterial para medir níveis de governança na UGRHI 05, no período entre 2013 e 2017. Os métodos utilizados são Programação por Compromisso (CP), Teoria dos Jogos Cooperativos (CGT), Método Analítico Hierárquico (AHP), ELECTRE II e PROMETHE II.

\section{GOVERNANÇA DOS RECURSOS HIIDRICOS}

A governança pública, como corrente da teoria da administração pública, procura compatibilizar os critérios de democratização com os de busca de melhor desempenho das políticas, reconhecendo o papel fundamental do Estado de liderar o processo de resolução dos problemas coletivos, mas reconhecendo também que deve fazê-lo a partir da interação com a sociedade (ABRUCIO et al., 2011 apud WWF BRASIL; FGV, 2014).

Para Zuffo e Zuffo (2016), a governança hídrica pode ser definida pelos sistemas políticos, sociais, econômicos e administrativos que afetam os usos, o desenvolvimento e a gestão dos recursos hídricos, referindo-se ainda a entrega ou serviços de água para diferentes usos e usuários, reforçando o que já foi mencionado pela FGV (2013), quando afirma que a governança envolve a gestão administrativa do Estado, assim como a capacidade de mobilizar e articular os diferentes atores sociais e estatais para resolver as ações coletivas sensíveis.

Na prática, espera-se que a governança da água determine quem fica com o quê, quando, como e quem tem o direito à água, aos serviços e aos benefícios correlatos, o que pressupõe o reflexo das aspirações da sociedade na tomada de decisões pela administração pública, e não a defesa de interesses específicos de determinados setores (ZUFFO; ZUFFO, 2016).

Já, o Global Water Partnership (2002, p. 1) define a governança das águas como "o conjunto de sistemas políticos, sociais, econômicos e administrativos disponíveis para aproveitar e gerenciar os recursos hídricos e, distribuir os serviços hídricos nos distintos níveis da sociedade".

Enfim, a diferença entre governança da água e gestão da água é que a governança é o conjunto de processos e instituições que definem e identificam quais são as metas de gestão a serem perseguidas. A gestão trata dos mecanismos e 
medidas práticas utilizadas para atingir as metas traçadas e, portanto, chegar a melhores resultados. Assim, a governança da água fornece a estrutura para decidir quais serão as atividades de gestão dos recursos hídricos que serão implementadas, o que leva a mencionar que uma crise de governança hídrica é uma crise nos processos de tomada de decisão e das instituições (LAUTZE, 2011 apud SANT'ANNA, 2012).

\section{MÉTODOS MULTICRITERIAIS}

De acordo com Keeney (1992 apud FANTINATTI, 2011), para resolução de problemas os métodos tradicionais buscam as soluções por alternativas e soluções ótimas, priorizando a racionalidade e ignorando a subjetividade dos envolvidos na tomada da decisão. Esses métodos não são capazes de lidar com as complexidades de problemas como as incertezas, os conflitos de valores e objetivos, diferenças na relação de poder entre os envolvidos, diversos critérios para a avaliação e até inexistência ou infinita quantidade de informações (FANTINATTI, 2011).

Ademais, o emprego de um único critério para tomar uma decisão mostra-se limitado, principalmente em questões que envolvam o meio ambiente. Por exemplo, analisar unicamente os critérios financeiros sobre as alternativas torna difícil quantificar outros aspectos, como os sociais e o paisagístico (MARTIN; LEGRET 2005).

Em contrapartida, os métodos multicriteriais permitem se valer de diferentes e variados pontos de vista sobre um processo de decisão, o que, por sua vez, cria situações conflituosas entre os diferentes atores, mas, ao mesmo tempo, permite a reflexão sobre uma gama maior de soluções possíveis. Roy e Bouyssou (1993 apud MARTIN; LEGRET, 2005) indicam que "no processo de auxílio à tomada de decisão, o objetivo principal não é descobrir uma solução, mas construir ou criar uma ferramenta que seja considerada útil aos olhos de um ator envolvido no processo de decisão".

Além disso, para Roy (1985 apud VENTURINI, 2003), os atores ou tomadores de decisão utilizam seus desejos, valores, interesses e preferências como base para seus julgamentos. Desse modo, é necessário buscar métodos que busquem equilibrar tais subjetividades humanas, bem como a assimetria de poder entre diferentes atores em torno de um problema.

Há inúmeros métodos multicriteriais, sendo possível agrupá-los em duas escolas: a Escola Americana, cuja solução é tida como a mais racional; e a Escola Europeia, a qual considera a solução de melhor compromisso.

Venturini (2003) exemplifica que a Escola Americana utiliza um modelo descritivista-prescritivista que se baseia em um ideal, em que as hipóteses buscam se aproximar ao máximo deste. Entretanto, a Escola Europeia usa um modelo construtivista, em que os atores constroem um modelo que evolui de acordo com seus valores, permitindo, assim, explorar fatores subjetivos geradores de recomendações que atendam às expectativas de tais atores.

Há diversas críticas à Escola Americana por focar seus objetivos no Tomador de Decisão (DM Decision Maker) e na procura da "Solução Ótima", o que poderia induzir ou impor um resultado ao DM. Por sua vez, a Escola Europeia preconiza a não existência de um problema isolado, bem como valoriza o que cada observador enxerga diante de um problema, estruturando o problema de acordo com seus valores. Além disso, não segrega os elementos subjetivos dos objetivos, e o decisor, conforme evolui no processo, pode modificar seus pontos de vista iniciais (ZUFFO et al. 2002).

Os diferentes pontos de vista, ao lidar com um problema de classificar, comparar e ordenar dife- 
rentes cenários, são importantes, pois permitem observar a presença ou não de uma determinada propriedade, reconhecer uma característica de valoração de um atributo qualitativo e atribuir uma nota para essa característica qualitativa (ROY, 1968).

Devido às variedades dos métodos multicriteriais, Figueira, Greco e Ehrgott (2005 apud FANTINATTI, 2011) e Zuffo (1998) categorizam-nos em 4 grandes grupos: 1) Métodos baseados na programação matemática (MOLP - Multiobjective Linear Programming); 2) Métodos baseados nas Teorias do Valor e da Utilidade Multiatributo (MAUT - Multiattribute Utilitary Theory); 3) Métodos baseados nas relações das aproximações hierárquicas e; 4) Abordagens não clássicas, como os que incorporam a matemática Fuzzy.

Cada método apresenta suas vantagens e desvantagens, sendo necessária a avaliação da adequação do modelo ao problema, bem como dos resultados apresentados. Também é recomendada a utilização de mais de uma ferramenta para comparação e confirmação dos resultados obtidos, o que por sua vez gera uma maior confiança ao DM na tomada de decisão.

\subsection{Escolha dos métodos}

Os modelos da Escola Europeia são os mais empregados em problemas com maior grau de complexidade, como os ligados à área ambiental, de recursos hídricos ou sanitária. Isso se deve principalmente à sua característica construtivista, que considera que o problema e as suas alternativas não estão prontos, necessitam ser construídos pelos atores envolvidos a partir de critérios objetivos e subjetivos, aumentando a compreensão destes sobre o problema e identificando novas e melhores alternativas, bem como as ações necessárias para sua implementação (FANTINATTI, 2011).
Apesar das críticas, alguns métodos da Escola Americana também são utilizados em problemas relacionados à área ambiental devido a sua flexibilidade e têm, no caso dos métodos multiplicativos, apresentado bons resultados quando comparados com os da Escola Francesa.

Gershon e Duckstein (1983) aplicaram o método ELECTRE da Escola Francesa, Programação por Compromisso (CP), Teoria dos Jogos Cooperativos (CGT) e a Teoria da Utilidade Multiatributo (MAUT), estes três últimos da Escola Americana, em um problema de planejamento da bacia hidrográfica do Rio Santa Cruz no Arizona. Os métodos apresentaram resultados similares, exceto o método ELECTRE que apresentou mais valores de discordância.

Outro caso foi o trabalho realizado por Zuffo et al. (2002), em que foram aplicados os Métodos ELECTRE II, PROMETHEE II, CP, CGT e o Método Analítico Hierárquico (AHP) para um problema de reabilitação, expansão e conservação do sistema produtor de água potável da Bacia do Cotia em São Paulo. Dos métodos utilizados, 4 apresentaram resultados praticamente idênticos, exceto o ELECTRE II, que obteve valores não satisfatórios dentro do cenário esperado. No entanto, Zuffo e Santos (2002) citam que essa falha ocorreu pois o método se mostra insensível a qualquer mudança dos pesos atribuídos aos critérios, propondo assim, duas alterações nas equações relativas à hierarquização, fazendo com que os resultados alcançassem valores mais satisfatórios.

Já Martin e Legret (2005) utilizaram o método ELECTRE para escolha de estruturas para a gestão das águas pluviais no meio urbano. Nesse trabalho foram enviadas enquetes para 524 organismos públicos e privados do Sudoeste da França que possuem suas atividades relacionadas com o tema. Como resultado, os autores perceberam que as alternativas apresentaram valores muito semelhantes, dificultando a sua hierarquização. 
O problema nos resultados foi atribuído ao fato de as alternativas serem altamente discriminativas, sobrepondo os pesos dos critérios. Portanto, recomendou-se a utilização de outros métodos para comparação.

Por fim, Orellana et al. (2018) aplicaram com sucesso o método CGT para identificar e classificar os setores elegíveis à reabilitação das redes de distribuição de água na zona norte da cidade de São Paulo. Um trabalho semelhante foi realizado por Trojan e Morais (2012), no qual os autores utilizam o método ELECTRE II com o mesmo objetivo, porém com seus critérios focados em alternativas de instrumentos para controle das perdas físicas e econômicas da rede.

\subsection{Métodos Utilizados}

No presente trabalho, adotaram-se 5 métodos diferentes das duas principais escolas, sendo os métodos CP, CGT e AHP pertencentes à Escola Americana. Já o ELECTRE II e o PROMETHEE II vinculam-se à Escola Europeia, que se referem à família dos métodos de aproximações hierárquicas. É relevante ressaltar que o AHP, nesse trabalho, foi utilizado apenas para fornecer um vetor de pesos que represente a importância dos diferentes critérios.

Paralelamente à utilização dos métodos anteriores, usou-se ainda a técnica DELPHI que, segundo Schmidt (1985), é aplicada para obtenção de informações de um grupo de especialistas, normalmente para atribuição de pesos aos critérios. Trata-se de um método subjetivo, não tendo, necessariamente, uma maior fundamentação matemática. Zuffo (2008) menciona que, para evitar efeitos negativos, como a dominação de determinados participantes, o método procura respostas escritas, normalmente formulários individuais.
Método Programação por Compromisso (Compromise Programming - CP): Zeleny (1982, apud ZUFFO; GENOVEZ, 2006) explica que o método é baseado no conceito de distância métrica (Teorema de Pitágoras) de dois pontos de coordenadas conhecidas. Assim, o método obtém a melhor solução como aquela que apresenta menor distância entre os pontos factíveis em relação a um "Ponto Meta" definido pelo DM (GERSHON; DUCKSTEIN 1983). O processo de definição desse "Ponto Meta" é facilitado ao restringi-lo como menor ou igual a um "Ponto Ideal" de tal modo que, muitas vezes, o "Ponto Ideal" é utilizado como "Ponto Meta" (Fig. 1).

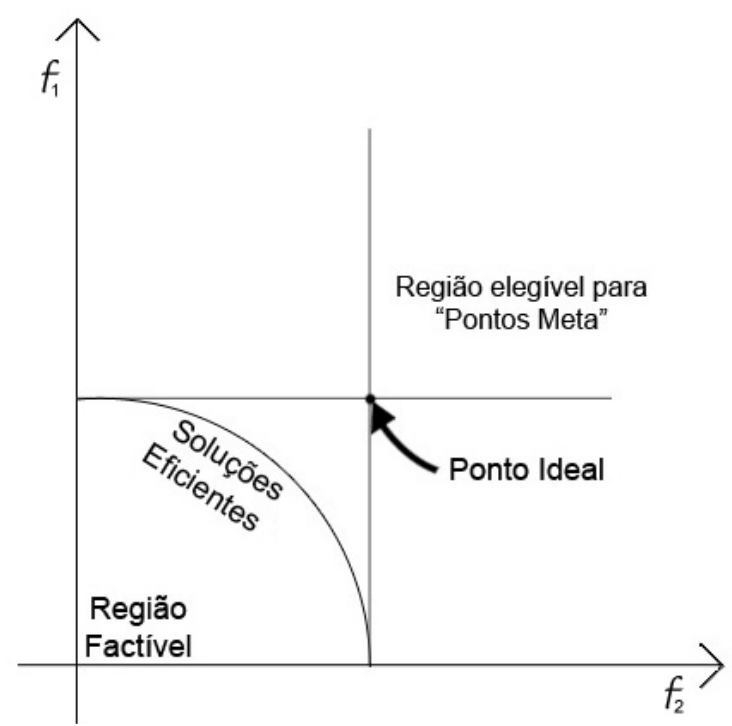

Figura 1 - Seleção do "Ponto Meta" Fonte: adaptado de Gershon e Duckstein (1983)

A distância é medida pela métrica $L_{-} p$, sendo definida como:

$$
L_{p}(x)=\left[\sum_{i=1}^{n} \alpha_{i}^{p}\left|\frac{f_{i}^{*}-f_{i}(x)}{f_{i}^{*}-f_{i, w}}\right|^{p}\right]^{1 / p}
$$

Zuffo et al. (2002) explicam que $f_{-}{ }^{\wedge}{ }^{\wedge *}$ é a função objetivo e a Solução Ideal é dada por sua maximização, logo: 


$$
f_{i}^{*}=\operatorname{Max}_{i}(x)
$$

Tendo o vetor $\mathrm{f}^{\wedge}$ *em que os elementos são todos maximizados:

$$
f^{*}=\left(f_{1}^{*}, f_{2}^{*}, \ldots, f_{n}^{*}\right)
$$

O autor ainda explica que, na prática, deve ser utilizada para a avaliação de soluções alcançáveis, visto que a obtenção da Solução Ideal não é possível, já que dificilmente existe um vetor de decisões $x^{*}$ que seja solução comum aos diferentes objetivos.

Os demais parâmetros da Eq. 1 são definidos como $\alpha_{i}$ como o peso dos critérios; $f_{i, w}$ o pior valor obtido pelo critério $i ; f_{i}(x)$ é o resultado da implementação da decisão com respeito ao ísimo critério; e p reflete a importância que o DM atribuiu aos desvios máximos. Para $p=1$, todos os desvios de $f_{i}^{*}$ são levados em consideração em relação a sua magnitude. Para $2<p<\infty$ os desvios máximos têm maior influência, já para $p=\infty$ o maior desvio é o único considerado (critério minimax). Gershon e Duckstein (1983) ressaltam que a Programação por Metas é análoga à Programação por Compromisso; todavia, o valor de $p$ é sempre igual a 1 e o "Ponto Meta" não é restrito.

\section{Método Teoria dos Jogos Cooperativos (Coo-} perative Game Theory - (GT): Nesse método a "Solução Ideal" também se baseia no cálculo da distância; todavia, a melhor solução é aquela que apresenta a maior distância de um determinado "Status Quo" - ponto de menor nível (GERSHON; DUCKSTEIN, 1983). Assim, a distância medida é a geométrica, dada por:

$$
g(x)=\prod_{i=1}^{n}\left|f_{i}(x)-f_{i}^{*}\right|^{\alpha_{i}}
$$

Os parâmetros $\alpha_{i}$ e $f_{i}(x)$ são os mesmos do método Programação de Compromisso, já $f_{i}^{*}$ representa o iésimo elemento do "Status Quo".

Zuffo et al. (2002) explicam que esse método matemático foi originado da teoria dos jogos (matemático americano John Forbes Nash Jr, 1928 - 2015), aplicável para resolução de conflitos, pois os participantes têm a oportunidade de se comunicar e fazer acordos.

Gershon e Duckstein (1983) destacam o desenvolvimento de 7 axiomas para obter uma solução cooperativa, no qual esses axiomas permitem aos atores envolvidos obter uma única solução, encontrada por meio de métodos padrões de uma programação não-linear. Apesar de apresentar uma única solução, qualquer solução intermediária é factível, uma vantagem dos métodos não-lineares. Outra vantagem do método é considerar que os objetivos trabalham conjuntamente em prol de uma melhor solução, ou seja, não há competição entre os objetivos sendo, portanto, a razão do seu uso em problemas que envolvam a área ambiental.

\section{Método Analítico Hierárquico (Analytic Hierarchy}

Process - AHP): A mente humana, ao se confrontar com um problema complexo, tende a decompor essa complexidade e, quando descobre relações, sintetiza-a. Tal modo de estruturar um problema serviu de base para Thomas L. Saaty desenvolver o AHP. A utilização desse processo permite reduzirsistemas extremamente intrincados a uma sequência de comparações aos pares de seus componentes. Essa sequência se dá por meio da decomposição do problema em diversos fatores, organizando-os em níveis hierárquicos. A hierarquia em questão é organizada com os objetivos finais no topo passando por sub-objetivos e limitadores e, terminando na base com os possíveis cenários.

Segundo Saaty (1977), um dos maiores desafios ao utilizar as teorias dos métodos de tomada de decisão é justamente atribuir pesos a cada um dos critérios. 
Logo, o método AHP tem como objetivo fornecer um vetor de pesos que represente a importância dos diferentes critérios. Isso é realizado por meio da comparação par-a-par entre os critérios já organizados de forma hierárquica, isto é, os de maior importância para os de menor importância (SCHMIDT, 1995).

O julgamento da análise dos DM é traduzido em uma escala numérica que varia de 1 a 9 de acordo com a relação de importância de um critério com o outro (Tabela 1).

Zuffo et al. (2002) explicam que a partir da análise par-a-par surge uma matriz de comparação $A=[n \times n]$, em que os seus elementos $s_{i, j}$ representam a superioridade do critério i em relação ao j, conforme equação abaixo:

$s_{i, j}=\frac{\alpha_{i}}{\alpha_{j}}$

Em que $\alpha_{\mathrm{i}(j)}$ é o peso para o critério $i(j)$.

Tabela 1 - A escala semântica de Saaty e sua correspondência numérica

\begin{tabular}{|l|c|}
\hline Escala Semântica & $\begin{array}{c}\text { Correspondência } \\
\text { numérica }\end{array}$ \\
\hline De igual importância & 1 \\
\hline De pequena importância sobre o segundo & 3 \\
\hline De grande importância sobre o segundo & 6 \\
\hline De importância muito grande sobre o segundo & 7 \\
\hline De importância absoluta sobre o segundo & 9 \\
\hline
\end{tabular}

Fonte: Fantinatti (2011)

Saaty (1977) ainda explica que a matriz é positiva e satisfaz a propriedade recíproca $s_{i, j}=1 / s_{i, j}$. Logo, essa matriz será multiplicada por sua transposta $\mathrm{W}$ obtendo o vetor $\lambda \mathrm{W}$. Assim, a matriz de comparação fornecerá o vetor de pesos dos n objetivos W. Logo o seu equacionamento fica:

$A W=\lambda W$

$$
(A-\lambda I) W=0
$$

Para avaliar o grau de consistência da matriz, Zuffo et al. (2002) indicam a determinação do coeficiente de consistência, conforme a equação:

$$
C=\frac{\left(\lambda_{\max }-n\right)}{(n-1)}
$$

Em que $\lambda_{\max }$ é o maior autovalor encontrado e $\mathrm{n}$ o número de critérios. Quanto mais próximo de zero for o valor de $\mathrm{C}$, mais consistente é a matriz.

O método AHP é comparável aos métodos da teoria multiatributo (MAUT) porque sua estrutura se assemelha a uma função aditiva. Desse modo, Benton (1986 apud ZUFFO et al., 2002) comparou o método AHP com outro que utiliza uma função aditiva simples conhecida por MAV (Multi-Attribute Value), do tipo:

$V_{i}=\sum_{j} \alpha_{j} x_{i j}$

Por fim, Zuffo et al. (2002) frisam a crítica de Benton ao método AHP em que este último afirma que as maiores fraquezas do método são a suposição da escala para a medida dos valores e a ambiguidade sobre os critérios de pesos, enquanto o método MAV se limita a critérios de nível simples.

Por sua vez, Saaty (1977) exalta que, no método AHP, a experiência e o conhecimento dos atores envolvidos no processo são, no mínimo, tão valiosos quantos os dados do problema.

Schmidt (1995) também defende o método AHP ao dizer que ele incorpora conhecimento e julgamentos permitindo articular, avaliar, debater e priorizar as questões envolvidas no problema. Desse modo, os julgamentos só serão obtidos por meio de um consenso entre os atores envolvidos na tomada de decisão. Assim, o AHP permite que tanto os critérios objetivos como os subjetivos sejam ordenados de uma maneira in- 
tuitiva e consistente, traduzindo de forma clara a preferência dos DM.

Método ELECTRE I e II: Pertencente à família da escola europeia, o método se baseia na relação hierárquica entre as diferentes alternativas. 0 Método ELECTRE I baseia sua escolha na alternativa que seja preferida pela maioria dos critérios e que, ao mesmo tempo, não seja inaceitável aos critérios analisados. Assim, para a aplicação do método, Roy (1968) desenvolveu três conceitos metodológicos, a saber: concordância, discordância e valores limites.

Tem-se a concordância entre duas alternativas $i$ e $j$ como a medida do número do critério para o qual a ação i é preferível em relação a $j$ ( $i$ P j) ou ambas são iguais $(i=j)$. Segundo Zuffo et al. (2002) o índice de concordância pode ser obtido, primeiramente, subdividindo os critérios em três subcritérios, sendo esses:

$I^{+}=I^{+}(i, j)-\{k \in I: i>j\} \quad$ para i preferível a j;

$I^{=}=I^{=}(i, j)-\{k \in I: i=j\} \quad$ quando i é equivalente $\mathrm{a} \mathrm{j}, \mathrm{e}$

$I^{-}=I^{-}(i, j)-\{k \in I: i<j\} \quad$ para j preferível a i.

Em que $k$ são todas as alternativas e $I$ todos os critérios. Após essa subdivisão, são determinadas as medidas dos pesos $(W)$ dos critérios por meio das equações como segue:

$$
\begin{aligned}
& W^{+}=\sum_{i \in I^{+}} \alpha_{i} \\
& W^{=}=\sum_{i \in I^{=}} \alpha_{i} \\
& W^{-}=\sum_{i \in I^{-}} \alpha_{i}
\end{aligned}
$$

Em que $\alpha_{i}$ os pesos atribuídos pelo decisores; vale ressaltar que, segundo Fantinatti (2001), esses pesos não mudarão durante a aplicação do método. De posse desses valores, o índice de concordância c(i,j) será definido pela Eq. 13:

$$
c(i, j)=\frac{W^{+}+\frac{1}{\text { fator }} W^{=}}{W^{+}+W^{=}+W^{-}}
$$

Sendo fator a relevância que o decisor arbitra para as medidas dos pesos de igual preferência $\left(W^{*}\right)$, podendo assumir valores iguais a 1 ou 2 , ou seja, $W^{=}$ terá a mesma ou a metade da relevância dos pesos de preferência positiva $\left(W^{+}\right)$, respectivamente.

Paralelo ao índice de concordância, há o índice de discordância que representa o grau de insatisfação em escolher uma determinada alternativa $i$ em relação a uma alternativa $j$. Para obtenção dessa medida é necessário, primeiramente, determinar uma escala comum para cada critério. Posteriormente se aplica a Eq. 14 para obtenção do índice de discordância $d(i, j)$.

$d(i, j)=\max _{k \in I^{-}} \frac{[z(i, k)-z(j, k)]}{R^{*}}$

Em que $z(i, k)$ e $z(j, k)$ é a avaliação da alternativa $i$ e $j$, respectivamente, segundo a escala normalizada para o critério $k$ e $R^{*}$, o maior valor das escalas numéricas de todos os critérios.

Por sua vez, os valores limites $(p, q)$ são obtidos a partir da relação hierárquica $(R)$, sendo essa última obtida das condições de concordância e discordância anteriormente apresentadas. 0 valor de $R$ é atribuído pelo DM e varia de zero a um, sendo que a alternativa $i$ é preferível a $j$ se $c(i, j)>p$ e $d(i, j)<q$. Consequentemente, o decisor deve escolher dois pares ( $p$ e $q$ ), que representam uma preferência forte e fraca, respectivamente e, desses pares, gerar dois gráficos distintos: o gráfico de preferência forte (GF) e o de preferência fraca (GF). A partir dos gráficos, 
Zuffo et al. (2002) indicam que se deva determinar o Kernel, que tem por objetivo representar as alternativas preferidas sobre a relação básica de ordenação.

O método ELECTRE II surgiu como extensão ao primeiro homônimo e utiliza os dois gráficos de preferência produzidos pelo ELECTRE I como dados de entrada. Zuffo (1998) indica que o método baseia sua escolha na alternativa que é preferida pela maioria dos critérios e, ao mesmo tempo, que não ultrapasse um nível de desconforto aceito pelo decisor. Assim, o ELECTRE II estabelece uma ordenação completa sobre um conjunto de alternativas de modo a satisfazer os testes de concordância e o de discordância.

Vale ressaltar que para o cálculo do índice de concordância c(i,j), o fator da Eq. 13 assume valor igual a 1, ou seja, os pesos de igual preferência $\left(W^{*}\right)$ têm a mesma relevância que os de preferência positiva $\left(W^{+}\right)$.

Por fim, o método procede à ordenação das alternativas em duas etapas preordenadas: classificação progressiva e classificação regressiva. Ambas as ordenações tendem a apresentar resultados diferentes, todavia um muito próximo do outro. Como resultado final, cabe ao DM decidir entre a média entre as duas classificações, conforme Eq. 15 ou, se os resultados não forem satisfatórios, redefinir o problema e reaplicar o método.

$$
m(x)=\frac{v^{\prime}+v^{\prime \prime}}{2}, \quad \forall x \in X
$$

Nessa etapa de hierarquização, Zuffo e Santos (2002) observam que o método apresenta uma falha por não relativizar as pequenas diferenças entre os valores de critérios no cálculo do índice de preferência. Desse modo, os autores propõem a aplicação de uma punição que altera a Eq. 15 como apresentado na Eq. 16:

$$
m(x)=\frac{v^{\prime}+v^{\prime \prime}}{2}+I C\left(\frac{\text { NoAlt }}{\operatorname{Min} \_O(x)+1}\right), \forall x \in X
$$

Em que NoAlt é o número de alternativas analisadas, Min_ $O(x)$ é a ordem da última alternativa e IC é o grau de inconsistência verificado entre as duas ordenações dado por:

$$
I C(x)=\left|v^{\prime}-v^{\prime \prime}\right|
$$

Método PROMETHEE II: Estabelece uma estrutura de preferência entre as alternativas, semelhantemente ao ELECTRE, assim Brans et al. (1986) citam que o processo é composto de duas etapas:

1. Construção das relações de preferências entre o grupo A de alternativas. É obtida pela definição de um índice e um gráfico de superação entre as alternativas, representando as preferências do decisor.

2. Avaliação dessas relações a partir do fluxo de entrada e saída do gráfico de superação das alternativas. A partir daí, o método PROMETHEE ॥ fornece uma ordenação completa de preferência das alternativas o que, por sua vez, auxiliará o decisor na solução do problema.

Para aplicação do método, Zuffo et al. (2002) apontam a necessidade das seguintes informações adicionais:

1. Informações entre critérios: representada pelos pesos $\alpha_{\mathrm{i}}$. Tais pesos são assumidos positivos e seus valores normalizados de forma que a sua soma seja igual a 1.

2. Informações internamente aos critérios: das comparações par-a-par das alternativas é observada a diferença entre os valores de um mesmo critério para diferentes alternativas e atribuído um valor de zero a um. 
Brans et al. (1986) citam que existe uma função de avaliação $f(a)$ para cada alternativa $a \in A$, e a comparação entre duas alternativas $a, b \in A$, é dada por uma função de preferência $P$ :

$$
P: A \times A \rightarrow(0,1)
$$

que representa a intensidade da preferência da alternativa a em relação a b como:

- $P(a, b)=0$ quando não há preferência de $a$ sobre $b$;

- $P(a, b) \sim 0$ para fraca preferência de $a$ sobre $b$;

- $P(a, b) \sim 1$ para forte preferência de $a$ sobre $b$;

$$
P(a, b)=\mathcal{P}(f(a)-f(b))
$$

- $P(a, b)=1$ quando há preferência estrita de $a$ sobre $b$.

Na prática, a função de preferência é dada pela diferença entre duas funções de avaliação, sendo assim a sua função descrita por:

Para cada critério $f$ é considerado um critério generalizado correspondente a uma função de preferência P. A maioria dos casos práticos é resolvida com seis tipos diferentes de critérios generalizados. Neste trabalho foi adotada a função de preferência gaussiana, que segue a função de preferência (Fig. 2).

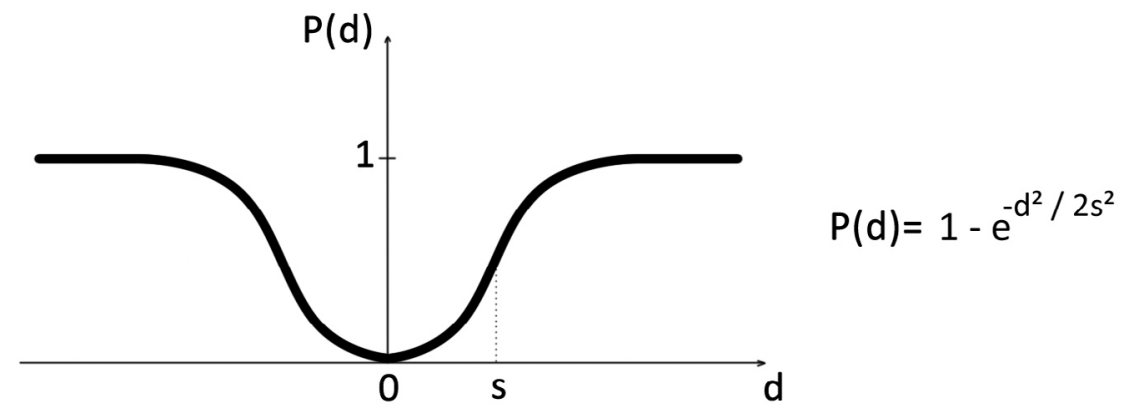

Figura 2 - Gráfico de função de preferência linear Fonte: adaptado de Brans et al. (1986)

O primeiro passo para aplicação do método é a definição dos índices de preferências agregados e os fluxos de hierarquização. Os índices agregados de preferências são dados pela Eq. 20:

$$
\pi(a, b)=\sum_{j=1}^{m} P_{j}(a, b) \alpha_{j}, \forall a, b \in A
$$

em que $\pi(a, b)$ expressa o grau em que ' $a$ ' é preferível a ' $b$ ' sobre todos os critérios e $\pi(b, a)$ expressa o grau em que ' $b$ ' é preferível a ' $a$ '. Zuffo et al. (2002) citam que ambos são positivos e devem respeitar as seguintes propriedades:

$$
\pi(b, a)=0 ; e, 0 \leq \pi(a, b) \leq 1 ; \quad \forall a, b \in A .
$$

Com o índice de preferência, é realizada a ordenação dos valores de preferência e, a partir dela, são estabelecidos os fluxos de importância. Segundo Fantinatti (2001), tais fluxos indicam como uma alternativa supera às demais alternativas (fluxo positivo), ou é superada em relação às demais alternativas (fluxo negativo). Respectivamente, os fluxos positivos e negativos são calculados pelas Eq. 21 e 22. 


$$
\begin{aligned}
\phi^{+}(a) & =\frac{1}{n-1} \sum_{x \in A} \pi(a, x) \\
\phi^{-}(a) & =\frac{1}{n-1} \sum_{x \in A} \pi(a, x)
\end{aligned}
$$

Consequentemente, a ordenação realizada pelo PROMETHEE se dá pelo fluxo de importância líquido dado por:

$$
\phi(a)=\phi^{+}(a)-\phi^{-}(a), \quad \forall a \in A
$$

A melhor alternativa será a que apresentar o maior valor na Equação 23. Brans et al. (1986) mencionam que a ordenação completa para o método PROMETHEE II é definida por:

$$
\left\{\begin{array}{lll}
a P_{I I} b & (' a \text { ' preferível a ' } b \text { ') } & \text { se } \phi(a)>\phi(b) \\
a I_{I I} b & \text { ('a' indiferente a ' } b \text { ') } & \text { se } \phi(a) \geq(b)
\end{array}\right.
$$

Por fim, estes últimos autores atentam que a ordenação completa do PROMETHEE II permite ao decisor encontrar uma resposta mais fácil a um problema, todavia a ordenação parcial do PROMETHEE I apresenta informações mais realistas.

\subsection{Definição dos critérios e subcritérios}

Os critérios, subcritérios e respectivos verificadores para avaliar a Governança dos Recursos Hídricos nas Bacias PCJ foram obtidos a partir de entrevistas com especialistas que possuem suas atividades ligadas diretamente à UGRHI 05. Por isso, tais critérios e subcritérios refletem diretamente os valores dos especialistas entrevistados. Após as entrevistas, elaborou-se um mapa cognitivo identificando os critérios e subcritérios para medição e verificação dos níveis de governança (Fig. 3).

Durante a conferência de decisão, alguns pontos levantados como importantes não foram eleitos como critérios por não apresentarem relação direta com o conceito de governança exposto neste trabalho ou por não serem mensuráveis objetivamente ou subjetivamente, inviabilizando a modelagem. Assim, ao término das rodadas de entrevistas e discussões, foram estabelecidos 5 critérios, 6 subcritérios e 8 verificadores que, a título de modelagem, significam 5 critérios e 8 subcritérios (Tabela 2).

Os dados foram coletados a partir de São Paulo (2011), Fundação Agência das Bacias PCJ (2013a, 2013b, 2014, 2015a, 2015b, 2016a, 2016b, 2017a, 2017b, 2018) e Comitês PCJ (2013a, 2013b, 2013c, 2015a, 2015b, 2015c, 2016, 2017a, 2017b, 2018a, 2018b), que correspondem às fontes de verificação. Enfatiza-se que o período analisado equivale a 5 anos, de 2013 a 2017, e que sua periodicidade foi anual, ou seja, para cada subcritério medido ou verificado há 5 alternativas levantadas, uma para cada ano. Portanto, alternativa 1 refere-se ao ano 2013, alternativa 2 ao ano 2014, alternativa 3 ao ano 2015, alternativa 4 ao ano 2016 e alternativa 5 ao ano 2017. 


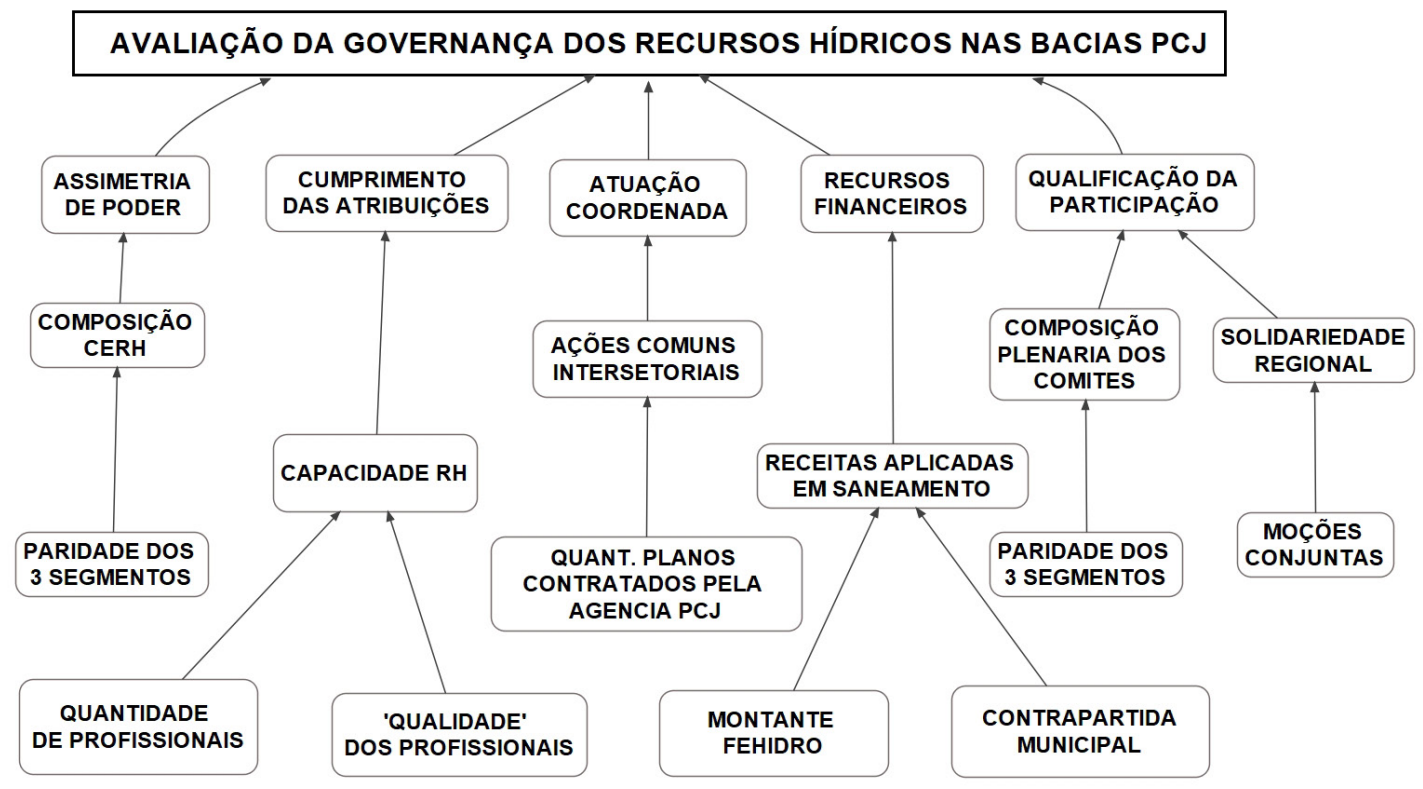

Figura 3 - Mapa cognitivo da avaliação da Governança nas Bacias PCJ

Tabela 2 - Descrição dos critérios, subcritérios e verificadores adotados

\begin{tabular}{|c|c|}
\hline Critérios & Subcritérios \\
\hline Assimetria de poder entre a RMSP e a região das bacias PCJ & $\begin{array}{l}\text { - Composição do Conselho Estadual de Recursos Hídricos de São Paulo - CRH } \\
\text { (Verificador: paridade na representação dos } 3 \text { segmentos) }\end{array}$ \\
\hline $\begin{array}{l}\text { Cumprimento das atribuições dos órgãos governamentais nos } \\
\text { Comitês PCJ }\end{array}$ & $\begin{array}{l}\text { - Capacidade dos recursos humanos do órgão executivo (Verificadores: quantidade e } \\
\text { qualificação) }\end{array}$ \\
\hline Atuação coordenada entre órgãos governamentais & $\begin{array}{l}\text { - Ações comuns intersetoriais - Recursos Hídricos e Saneamento (Verificador: } \\
\text { quantidade de planos municipais de saneamento básico contratados pela Agência PCJ) }\end{array}$ \\
\hline Recursos financeiros & $\begin{array}{l}\text { - Receitas aplicadas em saneamento nas bacias PCJ (Verificadores: Montantes do } \\
\text { FEHIDRO e dos Serviços Municipais de Água e Esgoto) }\end{array}$ \\
\hline \multirow[t]{2}{*}{ Qualificação da participação } & $\begin{array}{c}\text { - Composição da plenária dos Comitês PCJ (Verificador: paridade na representação dos } \\
3 \text { segmentos) }\end{array}$ \\
\hline & - Solidariedade regional (Verificador: existência de moções conjuntas entre segmentos) \\
\hline
\end{tabular}

Para aplicação dos modelos multicriteriais, é necessária a obtenção dos pesos de cada critério, em que o peso representa o grau de importância relativa que cada critério tem em relação aos demais. Assim, esses valores foram obtidos por meio de formulários individuais enviados para 7 especialistas que têm suas atividades em áreas relacionadas aos recursos hídricos, dos quais 5 foram respondidos, possibilitando assim a isenção de tendência e de influência na opinião de cada especialista.

$\mathrm{Na}$ sequência, aplicou-se um tratamento estatístico e matemático sobre as respostas e obtiveram-se três cenários de pesos, a saber: Cenário de pesos pelo AHP, em que as médias dos pesos atribuídos para cada critério foram posteriormente ajustadas pelo método AHP; cenário das médias, tomado apenas as médias simples das respostas; e cenário dos mínimos, no qual se utilizou como peso o menor valor atribuído pelos especialistas para cada critério, e consecutivamente sua escala foi normatizada pelo maior valor entre os mínimos. Adotou-se também uma escala de pesos entre a faixa de valores de 0 a 10, respectivamente, valores de menor e maior importância.

Com base nos resultados obtidos, é possível observar que, após o tratamento do método AHP, os pesos dos dois primeiros critérios obtiveram va- 
lores duas vezes maiores que os demais critérios (valor 10 contra máximo de 4,97), sendo que os valores atribuídos aos três últimos critérios os tornam irrelevantes para a avaliação da alternativa.

Já para os outros dois cenários de peso, a diferença entre o maior e o menor valor foi substancialmente menor, principalmente para o cenário de Média simples dos Pesos em que a maior diferença foi de 1,6 ponto.

\subsection{Nivel de desempenho e valoração das alternativas}

Os especialistas indicaram, por meio da resposta ao formulário encaminhado, o nível de desempenho desejado para cada critério e subcritério, ou seja, foi apontado para cada um destes o que representa o nível neutro ou o mínimo aceitável para determinado parâmetro e o nível bom ou aquele que corresponde ao nível desejável. Assim como nos pesos, foi padronizada uma escala de 0 a 10 para esses níveis, em que 0 representa o nível neutro e 10 o nível bom. Posteriormente, adotou-se um valor punitivo de -1 para o pior desempenho dentre os valores aquém do neutro, e uma bonificação de +1 (resultando no valor de 11) para o que apresentou o melhor valor entre os que superam o nível bom.

\section{RESULTADOS E DISCUSSÃO}

O estudo utilizou 4 métodos multicriteriais, como descrito anteriormente, a partir de uma Matriz "payoff" com os 3 cenários de pesos e os valores de desempenho de cada alternativa (Tabela 3). Destaca-se também a hierarquização completa de cada cenário de pesos (Tabelas 4 a 6).

Tabela 3 - Matriz de Avaliação

\begin{tabular}{|c|c|c|c|c|c|c|c|c|}
\hline \multirow{2}{*}{ Critérios } & \multicolumn{3}{|c|}{ Cenário de pesos } & \multicolumn{5}{|c|}{ Valores das alternativas } \\
\hline & AHP & Média & Min* & $A_{1}$ & $A_{2}$ & $A_{3}$ & $\mathrm{~A}_{4}$ & $A_{5}$ \\
\hline Recursos FEHIDRO & 10,00 & 8,40 & 10,00 & $-0,23$ & 0,19 & $-1,00$ & $-0,68$ & $-0,88$ \\
\hline Contrapartida Municipal & 10,00 & 8,40 & 10,00 & $-0,71$ & $-1,00$ & $-0,58$ & $-0,35$ & 1,51 \\
\hline Composição CRH & 4,97 & 8,00 & 8,57 & 0,00 & 0,00 & 0,00 & 0,00 & 0,00 \\
\hline Composição Comitês PCJ & 4,97 & 8,00 & 8,57 & $-0,74$ & $-0,74$ & $-1,00$ & $-1,00$ & $-1,00$ \\
\hline Capacidade RH - Qual. & 3,54 & 7,80 & 7,14 & 9,56 & 8,81 & 10,80 & 10,83 & 11,00 \\
\hline Ações Comuns Intersetoriais & 1,17 & 7,00 & 4,29 & 6,00 & 6,00 & 6,00 & $-1,00$ & $-1,00$ \\
\hline Capacidade RH - Quant. & 0,84 & 6,80 & 7,14 & 4,00 & 3,20 & 6,40 & 7,60 & 11,00 \\
\hline Solidariedade Regional & 0,84 & 6,80 & 5,71 & $-1,00$ & $-1,00$ & $-1,00$ & $-1,00$ & $-1,00$ \\
\hline
\end{tabular}

*Valores divididos pela décima parte do maior valor entre os mínimos obtidos entre os especialistas $(\div 0,7)$

Tabela 4 - Resultado da aplicação dos métodos multicriteriais ao cenário Média do Pesos corrigidos pelo método AHP

\begin{tabular}{|c|c|c|c|c|c|}
\hline \multirow{2}{*}{ Método } & \multicolumn{5}{|c|}{ Classificação das alternativas } \\
\cline { 2 - 6 } & $\mathbf{1}^{\mathbf{a}}$ & $\mathbf{2}^{\mathbf{a}}$ & $\mathbf{3}^{\mathbf{a}}$ & $\mathbf{4}^{\mathbf{a}}$ & $\mathbf{5}^{\mathbf{a}}$ \\
\hline CP (S= 1) & 5 & 1 & 3 & 2 & 4 \\
\hline CGT & 1 & 5 & 4 & 2 & 3 \\
\hline PROMETHEE II & 5 & 2 & 1 & 4 & 3 \\
\hline ELECTRE ॥ & 5 & 3 & 4 & 1 & \\
\hline
\end{tabular}

Tabela 5 - Resultado da aplicação dos métodos multicriteriais ao cenário Média dos Pesos

\begin{tabular}{|c|c|c|c|c|c|}
\hline \multirow{2}{*}{ Método } & \multicolumn{5}{|c|}{ Classificação das alternativas } \\
\hline & $1^{a}$ & $2^{a}$ & $3^{a}$ & $4^{a}$ & $5^{a}$ \\
\hline $\mathrm{CP}(\mathrm{S}=1)$ & 5 & 3 & 1 & 2 & 4 \\
\hline CGT & 1 & 2 & 3 & 5 & 4 \\
\hline PROMETHEE II & 5 & 3 & 1 & 2 & 4 \\
\hline \multirow{3}{*}{ ELECTRE ॥ } & \multirow{3}{*}{5} & \multirow{3}{*}{4} & 1 & & \\
\hline & & & 2 & & \\
\hline & & & 3 & & \\
\hline
\end{tabular}


Tabela 6 - Resultado da aplicação dos métodos multicriteriais ao cenário Pesos Mínimos

\begin{tabular}{|c|c|c|c|c|c|}
\hline \multirow{2}{*}{ Método } & \multicolumn{5}{|c|}{ Classificação das alternativas } \\
\hline CP $(\mathrm{S}=1)$ & $\mathbf{1}^{\mathbf{a}}$ & $\mathbf{2}^{\mathbf{a}}$ & $\mathbf{3}^{\mathbf{a}}$ & $\mathbf{4}^{\mathbf{a}}$ & $\mathbf{5}^{\mathbf{a}}$ \\
\hline CGT & 5 & 3 & 4 & 1 & 2 \\
\hline PROMETHEE II & 1 & 2 & 5 & 4 & 3 \\
\hline ELECTRE II & 5 & 1 & 4 & 3 & 2 \\
\hline & & & 1 & & \\
\hline
\end{tabular}

\subsection{Programação por Compromisso (CP)}

O método foi aplicado com base nos três cenários de pesos e utilizando o valor de $\mathrm{S}$, do equacionamento, igual a 1, permitindo uma comparação em igualdade com os demais métodos multicriteriais.

Nota-se que os resultados para os diferentes cenários de peso apresentam a Alternativa 5 (ano de 2017) como sendo a de melhor nível de desempenho de governança. Já a Alternativa 4 (ano de 2016) foi a pior, exceto para o cenário de Pesos Mínimos, no qual a Alternativa 2 foi a pior. Os cenários do método AHP e dos Pesos Médios apresentaram uma hierarquização muito próxima, havendo apenas uma alteração entre a segunda e terceira posição.

O cenário de pesos mínimos foi o que apresentou uma hierarquização mais discordante em relação aos demais. Essa diferença ocorre principalmente porque há uma mudança da ordem de importância (pesos) de determinados critérios em comparação aos demais cenários. Outro fator que dificultou um melhor desempenho do método é o fato das notas dos critérios apresentarem pouca diferença entre as alternativas, impactando diretamente nos resultados de preferência, sendo que, algumas vezes, a ordem de preferência foi decidida na terceira casa decimal.

\subsection{Teoria dos Jogos Cooperativos (CGT)}

Da análise da hierarquização é observada uma maior sensibilidade à mudança dos pesos, demonstra- da pela grande diferença entre as preferências na comparação entre cenários do método. Do mesmo modo, o Método CGT apresentou uma hierarquização das alternativas discordante com os outros métodos, pois é possível observar que a Alternativa 1 aparece como a melhor em todos os cenários de peso, sendo que nos demais métodos a Alternativa 5 foi escolhida unanimemente como a melhor.

Assim, além de o método ser extremamente sensível a variações de peso, ele não apresentou resultados satisfatórios.

\subsection{PROMETHEE II}

A modelagem utilizando o método PROMETHEE II, assim como as demais, apresentou resultados divergentes em comparação aos outros cenários, indicando também ser um método sensível a alteração de peso. Apesar disso, a Alternativa 5 foi apontada como de melhor desempenho em todos os cenários estudados. Nas demais posições de preferência, dependendo do critério de pesos houve uma grande diferença da hierarquização. Mesmo assim, o cenário de pesos médios apresentou hierarquização idêntica ao método CP.

Neste cenário, a segunda melhor alternativa é a 3 , e a de pior desempenho é a Alternativa 4, sendo que para o cenário de pesos AHP a Alternativa 3 é a de pior desempenho. Essa alteração é prova do impacto da mudança dos pesos dos critérios, principalmente para as alternativas que apresentam notas de desempenho muito próximas.

\subsection{ELECTRE II}

O método ELECTRE II apresentou uma melhor hierarquização após a correção obtida pelo cálculo de inconsistência. Como nos demais métodos, a Alternativa 5 foi apontada como a de melhor desempenho em todos os cenários estudados. Em segundo lugar no cenário de pesos mínimos e médios está a 
Alternativa 4; já para o cenário de pesos corrigido pelo AHP aparece a Alternativa 3 nesta posição.

Apesar da utilização da correção proposta, nas últimas posições houve um empate entre as Alternativas 1, 2 e 3 no cenário de pesos mínimos e médios, e entre a 1 e 2 para o cenário AHP. Assim, o método se mostra pouco sensível a mudança de pesos, mas, devido ao grande número de empates, demonstra-se que o método ainda não consegue oferecer uma boa hierarquização em critérios com notas muito próximas.

\section{CONCLUSÕES}

A análise multicriterial empregada para avaliação da Governança dos Recursos Hídricos nas Bacias PCJ permitiu abranger diferentes visões sobre o problema e possibilitou aos envolvidos no processo conhecer detalhadamente a governança do sistema na UGRHI 05.

Com exceção do método CGT, a Alternativa 5 (ano de 2017) foi escolhida como a de melhor desempenho independentemente do critério de peso adotado. Todavia, não foi possível perceber uma tendência de preferência nas demais posições, pois os métodos apresentaram muita divergência entre si e nos diferentes cenários de peso. $\mathrm{A}$ única exceção se dá entre o método $\mathrm{CP} \mathrm{e}$ PROMETHEE II no cenário de pesos médios que apresentaram a mesma hierarquização.

O método CGT teve seus resultados considerados insatisfatórios por apresentar uma hierarquização mais divergente e, principalmente, por indicar uma preferência por uma alternativa totalmente diferente dos demais métodos. Assim, tais resultados não serviriam como base para uma tomada de decisão.

O método PROMETHEE II apresentou resultados satisfatórios ao se comparar aos demais métodos, mas mostrou-se muito sensível a mudança de pe- sos. Por sua vez, o método ELECTRE apresentou bons resultados em comparação aos demais métodos, além de ter se mostrado menos sensível a mudança de pesos, já que sua hierarquização se deu muito semelhante nos três cenários de peso. 0 método CP também apresentou resultados coerentes em comparação aos demais métodos e mostrou pouca sensibilidade à mudança de pesos, ainda que um pouco maior que o método ELECTRE.

Assim, os métodos da Escola Francesa e o método (CP) da Escola Americana foram os que apresentaram resultados que serviriam como auxílio à tomada de decisão. Vale ressaltar que, neste estudo, o cenário de pesos mínimos altera a ordem de importância de determinados critérios, resultando na diferença de hierarquização nas últimas posições.

Observa-se ainda que, devido às características do tema em estudo e seus critérios de avaliação, as alternativas apresentam notas de avaliação muito próximas, o que colaborou na diferença de hierarquização de acordo com a sensibilidade de cada método. Do mesmo modo, as alternativas apresentaram valores de desempenho insatisfatórios para seus critérios de avaliação, por isso a escala padronizada dos níveis de desempenho necessitaria de mais valores de referência para a punição. Recomenda-se então que os especialistas adotem uma escala negativa maior para os valores insatisfatórios.

Com os resultados também é possível concluir que ao se aplicar as ferramentas de auxílio à tomada de decisão, os atores envolvidos devem utilizar mais de um método, visando garantir uma maior segurança e confiança aos tomadores de decisão.

Do mesmo modo, a definição dos pesos e importância dos critérios parte de fatores, muitas vezes subjetivos e ambíguos, o que demonstra a importância dos atores chave para os métodos de auxílio à tomada de decisão.

Portanto, recomenda-se mais de uma rodada de discussões para atribuição de pesos e de níveis 
de desempenho para argumentações e possíveis ajustes em tal atribuição por parte dos 5 especialistas que responderam ao formulário específico. Isso proporciona a cada um compreender os outros pontos de vista existentes, promove adequações a partir desse novo entendimento e facilita propostas de soluções mais viáveis.

Logo, um processo mais amplo para aplicação da análise multicriterial com atores relevantes na gestão dos recursos hídricos das bacias PCJ pode e deve englobar evento específico com um público-alvo maior, objetivando construir um mapa cognitivo referente à estruturação do problema e à definição dos critérios, subcritérios, indicadores e/ou verificadores, após alinhamento a respeito de conceitos importantes, como governança, análise multicriterial e seus modelos. $\mathrm{O}$ aprimoramento na definição dos critérios e subcritérios a serem avaliados e na atribuição dos pesos e dos níveis em questão resulta em uma aplicação mais adequada dos modelos de auxílio à tomada de decisão utilizados em tal artigo.

\section{AGRADECIMENTOS}

O primeiro autor agradece ao Conselho Nacional de Desenvolvimento Científico e Tecnológico (CNPq) pela bolsa concedida. O segundo autor agradece à Coordenação de Aperfeiçoamento de Pessoal de Nível Superior (Capes) pela bolsa concedida. Os autores agradecem a Luiz Roberto Moretti (Comitês PCJ), Sérgio Razera (Agencias da Bacia PCJ), Maíra Simões Cucio e Marcos Paulo Lallo Sartori pelas entrevistas concedidas.

\section{CONTRIBUIÇÃO DOS AUTORES}

Conceitualização: Rando AS e Luz DL; Metodologia: Luz DL, Rando AS e Zuffo AC; Investigação: Rando AS e Luz DL; Redação - Primeira versão: Luz DL e Rando AS; Redação- Revisão \& Edição:
Zuffo AC, Ferrão AMA, Luz DL e Rando AS; Supervisão: Zuffo $A C$ e Ferrão AMA.

\section{REFERÊNCIAS}

BENTON, V. A comparison of the analytic hierarchic process and simple multiattibute value function. European Journal of Operation Research, 26. p 7-21, 1986. apud ZUFFO, A. et al. Aplicação de Métodos Multicriteriais ao Planejamento de Recursos Hídricos. Revista Brasileira de Recursos Hídricos, v. 7, n. 1, p. 81-102, 2002.

BRANS, J. P. et al. How to select and how to rank projects: The Promethee method. European Journal of Operational Research, v. 24, n. 2, p. 228-238, fev. 1986. https://doi.org/10.1016/03772217(86)90044-5

COMITÊ DAS BACIAS HIDROGRÁFICAS DOS RIOS PIRACICABA, CAPIVARI E JUNDIAÍ (2018). CBH-PC): membros. Piracicaba: CBH-PCJ. Disponível em: <http://www.comitespcj.org.br/index. php?option=com_content\&view $=$ article\&id $=256 \& \mid$ temid $=354>$. Acesso em: 21 nov. 2018.

COMITÊS DAS BACIAS HIDROGRÁFICAS DOS RIOS PIRACICABA, CAPIVARI E JUNDIAÍ. Deliberação dos Comitês PCJ no 166/2013, de 27/03/2013. Empossa representantes dos órgãos governamentais (municípios, estados e União) e da sociedade civil (Usuários de Recursos Hídricos e Organizações Civis) nos Plenários do CBH-PC], do PCJ FEDERAL, para o mandato 2013 a 2015, e do CBH-PJ mandato 2013 a 2017. Disponível em: <http://www. comitespcj.org.br/images/Download/DelibComitesPCJ166-13. pdf>. Acesso em: 21 nov. 2018.

Deliberação dos Comitês PCJ no 167/2013, de 27/03/2013. Elege e empossa dirigentes dos Comitês PCj; define os municípios (SP e MG) com direito a voto, define a Secretaria Executiva; elege representantes dos municípios paulistas no CRH-SP e CMC-SP; indica representantes dos Comitês PCJ nos Fóruns Paulista, Mineiro e Nacional de Comitês de Bacias e na Rede Brasil de Organismos de Bacias Hidrográficas - REBOB e indica os representantes para compor a CT-PL, para o mandato 2013/2015 do CBH-PC) e para o mandato 2013/2017 do CBH-PJ, e dá outras providências. Disponível em: <http://www.comitespcj. org.br/images/Download/DelibComitesPCJ167-13.pdf>. Acesso em: 21 nov. 2018.

Deliberação dos Comitês PCJ no 190/2013, de 07/11/2013. Aprova manifestação sobre a renovação da outorga do Sistema Cantareira, em 2014, e dá outras providências. Disponível em: <http://www.comitespcj.org.br/images/ Download/DelibComitesPCJ190-13.pdf>. Acesso em: 24 nov. 2018.

Deliberação dos Comitês PCJ no 217/2015, de 27/03/2015. Empossa representantes dos órgãos governamentais (municípios, estados e União) e da sociedade civil (Usuários de Recursos Hídricos e Organizações Civis) nos Plenários do CBH- 
PCJ e do PCJ FEDERAL, para o mandato 2015 a 2017, e dá outras providências. Disponível em: <http://www.comitespcj. org.br/ images/Download/DelibComitesPCJ217-15.pdf>. Acesso em: 21 nov. 2018.

Deliberação dos Comitês PCJ no 218/2015, de 27/03/2015. Elege e empossa dirigentes dos Comitês PC); define os municípios (SP e MG) com direito a voto, define a Secretaria Executiva; elege representantes dos municípios paulistas no CRH-SP; indica representantes dos Comitês PCJ nos Fóruns Paulista, Mineiro e Nacional de Comitês de Bacias e na Rede Brasil de Organismos de Bacias Hidrográficas - REBOB e indica os representantes para compor a CT-PL, para o mandato 2015/2017, e dá outras providências. Disponível em: <http://www.comitespcj. org.br/images/Download/DelibComitesPCJ218-15.pdf>. Acesso em: 21 nov. 2018.

Deliberação dos Comitês PCJ no 234/2015, de 12/08/2015. Aprova manifestação sobre a renovação da outorga do Sistema Cantareira, em 2015, e dá outras providências. Disponível em: <http://www.comitespcj.org.br/images/ Download/DelibComitesPCJ234-15.pdf>. Acesso em: 24 nov. 2018.

Deliberação dos Comitês PCJ $n^{\circ}$ 252/2016, de 24/06/2016. Aprova manifestação sobre a renovação da outorga do Sistema Cantareira, em 2016, e dá outras providências. Disponível em: <http://www.comitespcj.org.br/images/ Download/DelibComitesPCJ252-16.pdf>. Acesso em: 24 nov. 2018.

Deliberação dos Comitês PCJ no 263/2017, de 31/03/2017. Empossa representantes dos órgãos governamentais (municípios, estados e União) e da sociedade civil (Usuários de Recursos Hídricos e Organizações Civis) nos Plenários do CBH-PC) e do PCJ FEDERAL, para o mandato 2017 a 2019, e dá outras providências. Disponível em: <http://www. comitespcj. org.br/images/Download/DelibComitesPCJ263-17. pdf>. Acesso em: 21 nov. 2018.

Deliberação dos Comitês PCJ no 264/2017, de 31/03/2017. Elege e empossa dirigentes dos Comitês PCj; define os municípios (SP e MG) com direito a voto, define a Secretaria Executiva; elege representantes dos municípios paulistas no $\mathrm{CRH}$ SP, CMC-SP e CONESAN-SP; indica representantes dos Comitês PCJ nos Fóruns Paulista, Mineiro e Nacional de Comitês de Bacias e na Rede Brasil de Organismos de Bacias Hidrográficas - REBOB e indica os representantes para compor a CT-PL, para o mandato 2017/2019, e dá outras providências. Disponível em: <http://www. comitespcj.org.br/images/Download/DelibComitesPC)264-17. pdf>. Acesso em: 21 nov. 2018.

(2018). Moções PCJ. Piracicaba: Comitês PCJ. Disponível em: <http://www.comitespcj.org.br/index.php?option=com_ content $\&$ view $=$ article $\& i d=168 \& \mid t e m i d=230>$. Acesso em: 24 nov. 2018.
(2018). PCJ-Federal: membros. Piracicaba: PCJ Federal. Disponível em: <http://www.comitespcj.org.br/index. php?option=com_content\&view $=$ article $\&$ id $=257 \&$ Itemid $=355>$. Acesso em: 21 nov. 2018.

FANTINATTI, P. A. P. Abordagem MCDA como ferramenta de mudanças de paradigmas no planejamento dos recursos hídricos. Tese (Doutorado em Engenharia Civil) - Faculdade de Engenharia Civil, Arquitetura e Urbanismo, Universidade Estadual de Campinas, Campinas. 399p. 2011.

FIGUEIRA, J.; GRECO, S.; EHRGOTT, M. (Ed.) Multiple criteria decision analysis: state ofart surveys. Boston, USA: Springer Science, Business Media. 2005 apud FANTINATTI, P. A. P. (2011) Abordagem MCDA como ferramenta de mudanças de paradigmas no planejamento dos recursos hídricos. Tese (Doutorado em Engenharia Civil) - Faculdade de Engenharia Civil, Arquitetura e Urbanismo, Universidade Estadual de Campinas, Campinas. 399p. 2011.

FUNDAÇÃO AGÊNCIA DAS BACIAS HIDROGRÁFICAS DOS RIOS PIRACICABA, CAPIVARI E JUNDIAÍ (2013b). Relatório anual de acompanhamento das ações executadas com recursos das Cobranças Federal, Estadual Paulista e Fundo Estadual de Recursos Hídricos 2013. Piracicaba: Agência das Bacias PCJ.

(2014). Relatório anual de acompanhamento das ações executadas com recursos das Cobranças Federal, Estadual Paulista e Fundo Estadual de Recursos Hídricos 2014. Piracicaba: Agência das Bacias PCJ.

(2015b). Relatório anual de acompanhamento das ações executadas com recursos das Cobranças Federal, Estadual Paulista e Fundo Estadual de Recursos Hídricos 2015. Piracicaba: Agência das Bacias PCJ.

(2016b). Relatório anual de acompanhamento das ações executadas com recursos das Cobranças Federal, Estadual Paulista e Fundo Estadual de Recursos Hídricos 2016. Piracicaba: Agência das Bacias PCJ.

(2017b). Relatório anual de acompanhamento das ações executadas com recursos das Compensações Financeiras I Royalties, Cobranças Federal e Estadual Paulista 2017. Piracicaba: Agência das Bacias PCJ.

(2017a). Relatório de gestão das bacias PCJ 2016. Piracicaba: Parla.

(2018). Relatório de gestão das bacias PCJ 2017. Piracicaba: Agência das Bacias PCJ.

(2015a). Relatório de gestão e situação das bacias PCJ 2014. Valinhos: Gráfica e Editora Discopel.

(2016a). Relatório de gestão e situação das bacias PC] ano base 2015. Piracicaba: Parla. 
(2013a). Relatório sobre a execução do Primeiro Termo Aditivo do Contrato de Gestão no 003/ANA/2011 Bacias PCJ. Piracicaba: Agência das Bacias PCJ.

FUNDAÇÃO GETÚLIO VARGAS - FGV (2013). A governança do sistema nacional de gerenciamento de recursos hídricos: diagnóstico e caminhos para seu aperfeiçoamento. São Paulo: FGV. (Relatório Final).

GERSHON, M.; DUCKSTEIN, L. Multiobjective Approaches to River Basin Planning. Journal of Water Resources Planning and Management, v. 109, n. 1, p. 13-28, jan. 1983. https://doi. org/10.1061/(ASCE)0733-9496(1983)109:1(13)

GLOBAL WATER PARTNERSHIP. Dialogue on effective water governance: learning from the dialogues. GWP. Stockholm, Sweden. Disponível em: http://www.waterinfo.gr/pages/ GWPfolderGovernance.pdf. 2002. Acesso em: 19 out. 2014.

KEENEY, R. L. Value-focused thinking: a path to creative decisionmaking. Cambridge: Harvard University Press, 1992 apud FANTINATTI, P. A. P. (2011) Abordagem MCDA como ferramenta de mudanças de paradigmas no planejamento dos recursos hídricos. Tese (Doutorado em Engenharia Civil) - Faculdade de Engenharia Civil, Arquitetura e Urbanismo, Universidade Estadual de Campinas, Campinas. 399p. 2011.

MARTIN, C.; LEGRET, M. Définitions, principe et exemple d'application à la gestion des eaux pluviales en milieu urbain. Bulletin des laboratoires des ponts et chaussées, n. 258-259, 2005, p. 29-46, 2005.

ORELLANA, A. et al. Contribuição ao planejamento de reabilitação de redes de distribuição de água. Ribagua - Revista Iberoamericana del Agua, p. 1-13, out. 2018. https://doi.org/10. 1080/23863781.2018.1495991

ROY, B. Classement et choix en présence de points de vue multiples (la méthode ELECTRE). Revue française d'informatique et de recherche opérationnele, TOMO II. v. 1, p.57-75, 1968. https:// doi.org/10.1051/ro/196802V100571

Méthodologie Multicritère d’Aide à la Décision. Ed. Economia, Paris, 1985 apud VENTURINI, M. A. A. G. Metodologia de Análise e Decisão Multicriterial Para a Reabilitação de Sistemas de Abastecimento de Água. Tese (Doutorado em Engenharia Civil) - Faculdade de Engenharia Civil, Arquitetura e Urbanismo, Universidade Estadual de Campinas, Campinas. 289p, 2003.

; BOUYSSOU D. Aide Multicritère à la Décision: Méthodes et Cas, Économica, Paris. 695 p. 1983 apud MARTIN, C.; LEGRET, $M$. Définitions, principe et exemple d'application à la gestion des eaux pluviales en milieu urbain. Bulletin des laboratoires des ponts et chaussées, n. 258-259, 2005, p. 29-46, 2005.

SAATY, T. L. A scaling method for priorities in hierarchical structures. Journal of Mathematical Psychology, v. 15, n. 3, p. 234-281, 1977. https://doi.org/10.1016/0022-2496(77)90033-5
SANT'ANNA, F. M. Tensões e conflitos na governança dos recursos hídricos amazônicos transfronteiriços. GEOUSP Espaço e Tempo, São Paulo, n. 31, p. 132-145, 2012. https://doi. org/10.11606/issn.2179-0892.geousp.2012.74275

SÃO PAULO (Estado). Decreto No 57.113, de 7 de julho de 2011. Adapta o Conselho Estadual de Recursos Hídricos - CRH e o Comitê Coordenador do Plano Estadual de Recursos Hídricos - CORHI, criados pelo Decreto $n^{\circ} 27.576$, de 11 de novembro de 1987, às disposições da Lei nº 7.663, de 30 de dezembro de 1991.

SCHMIDT, A. M. A. Processo de Apoio à Tomada de Decisão Abordagens: AHP e MACBETH. Dissertação (Mestrado em Engenharia de Produção) - Departamento de Engenharia de Produção e Sistemas, Universidade Federal de Santa Catarina, Florianópolis. 155p, 1995.

TROJAN, F.; MORAIS, D. Prioritizing alternatives for maintenance of water distribution networks: A group decision approach. Water SA, v. 38, n. 4, 2012. https://doi.org/10.4314/wsa.v38i4.11

VENTURINI, M. A. A. G. Metodologia de Análise e Decisão Multicriterial Para a Reabilitação de Sistemas de Abastecimento de Água. Tese (Doutorado em Engenharia Civil) - Faculdade de Engenharia Civil, Arquitetura e Urbanismo, Universidade Estadual de Campinas, Campinas. 289p., 2003.

WWF BRASIL; FUNDAÇÃO GETÚLIO VARGAS. Governança dos recursos hídricos: proposta de indicador para acompanhar sua implementação. São Paulo: WWF Brasil e FGV. ISBN 978-8586440-85-4, 2014.

ZELENY, M. -Multiple Criteria Decision Making. McGraw-Hill Book Company. New York. 536 p. 1982 apud ZUFFO, A. C.; GENOVEZ A. M. Método Multricriterial Utilizado Como Indicador da Qualidade de Água. XXII Congreso Latino Americano de Hidráulica. Ciudad Guayana, Venezuela. 11 p., 2006.

ZUFFO, A. C. "O processo Delphi”. in Avaliação multicriterial em tomada de decisão. Notas de aula. Programa de Pós-Graduação em Engenharia Civil (Área de Concentração: Recursos Hídricos), Faculdade de Engenharia Civil, Arquitetura e Urbanismo, Universidade Estadual de Campinas, Campinas-SP, 2008.

et al. Aplicação de Métodos Multicriteriais ao Planejamento de Recursos Hídricos. Revista Brasileira de Recursos Hídricos, v. 7, n. 1, p. 81-102, 2002. http://dx.doi.org/10.21168/rbrh.v7n1. p81-102

; SANTOS R. F. Método Electre II Aplicado ao Planejamento de Recursos Hídricos: Uma Proposta Para Melhorar a Performance do Método. XX Congreso Latino Americano de Hidráulica. Havana, Cuba. 10 p., 2002.

; ZUFFO, M. S. R. Gerenciamento de recursos hídricos: conceituação e contextualização. Rio de Janeiro: Elsevier, 2016. 